\title{
Editorial: Re-wiring Electronic Music
}

A new community of 'makers' has emerged in electronic music. These makers are not content with using off-theshelf 'instruments', but seek to create their own devices and systems to generate sound. Initially this would seem to have transpired through the need to personalise and critique our relationship with ever increasing esoteric and corporate technologies. There is a focus on the handmade, a DIY (do-it-yourself) ethos, working directly with materials and craft, raw analogue and hybrid electronics, and found sound and noise.

But the electronics are only part of a bigger picture. Doing it yourself requires knowledge, and inadvertently this has created forums to exchange and share ideas and information. It is more a case of DIT (doing-it-together). The Internet has provided a depository of 'how-tos', whilst the workshop has physically brought like-minded people together from a range of disciplines to foster a new artistic practice. The boundaries between instrument making, composing and performing have become increasingly blurred to reveal an overriding process and participatory approach that is as much about sociopolitical concerns as electronic music. The bringing together of practitioners into a realm of shared experiences has highlighted the importance of collective music making and has encouraged the rise of the large electronic music ensemble. This in-the-momentness has also placed an emphasis on the live as opposed to the recording. By starting from the ground up, in the very essence of how a sound is made, there is also a predisposition towards exploration and invention resulting in a truly experimental practice.

Running parallel with the ephemerality of the live performance, shared experiences and sound making are the physical things that are left behind: sound artefacts, instruments, circuits and assemblages where sound and the plastic arts meet. These devices then take the form of musical scores or documents of the artistic process, or works of art in their own right.

The issue begins with an article by Andrea Valle who discusses his Rumentarium, a computer-based sound generating system involving physical objects as sound sources excited by DC motors. Valle's system explores working with computer code and voltages as well as electroacoustic and electromechanical ways of generating sound. $\mathrm{He}$ is concerned with how composition and instrument design overlap to produce music. Valle also views how the system can be 'played' like an instrument through the addition of MIDI controllers or driven by algorithmic strategies.
The idea of 'softening' hardware through the use of microcontrollers is also discussed. The works of Kanta Horio, Zimoun, and John Bowers and Phil Archer amongst others are cited as points of reference.

Morten Riis' Steam Machine Music provides a catalyst for the investigation of older technologies to provide new knowledge and understanding to rudimentary conditions regarding electronic music. These ideas are placed in context of the growing field of media archaeology. Riis' work focuses on material physicality and the notion of operative technology as a basis for electronic music. He discusses in detail the construction of his steam-powered mechanical instrument that is built mostly from vintage Meccano parts and where the majority of sounds are created independently of electricity. Riis also considers the 'machine' itself as proving a document of the music.

Sanne Krogh Groth continues the discussion on the work of Riis alongside the Danish composer/ artist Goodiepal. Groth argues that both composers, through their self-constructed instruments, challenge the broader conventions of computer music. She also suggests that theories from the field of media archaeology can be used to provide a greater understanding and insight into the area of DIY electronic music. Groth looks carefully at how Riis operates on a micro-level exploring the functions and dysfunctions of media, whilst Goodiepal's macro-level approach deals in the realm of imaginary media.

My own article in this issue puts forward the question: 'With the first decade of the twenty-first century over and the DIY ethos firmly established to the point of becoming mainstream, what developments, if any, in this approach have occurred?' The shift in emphasis from DIY to DIT (do-it-together) is discussed along with the musical consequences of collective instrument building, and large group and delegated performance. The concept of extended musical process through building sound devices and instruments is highlighted with some specific examples of my work. The article raises further questions regarding the definition of musical instrument and also presents the idea of physical artefact as musical document. The rise of the DIY expert and the boutique synthesiser are also discussed with particular reference to Martin Howse, Tristan Perich, Tatsuya Takahashi and the group FM3.

Kazuhiro Jo, Adam Parkinson and Atau Tanaka present 'workshopping' as a collective creative musical practice. Central to their thesis is the notion of 'Music 
One Participates In' where there is a focus on a shift from listener-as consumer to participant-actor actively engaged in sound perception and production. The article also discusses workshop theory and Arnstein's ladder of citizen participation. The authors conclude with a range of reports on their own work that include the Mobile Music Workshop, How to Turn Your iPhone into a Musical Instrument, which introduces an interactive music programming and performance environment to non-specialists; Chiptune Marching Band, self-powered analogue circuits for street performance; Aeolian Harp, where ideas of generative music are explored; and Social Inclusion through the Digital Economy, a large multidisciplinary project covering areas of health, transport, home and business, alongside working with digital natives and mobile technologies.

The appropriation of older paradigms in technology that are then placed in modern contexts is the main theme of Lorin Edwin Parker's article. Parker presents a detailed case study of his instrument the Phantastron: a DIY vacuum tube instrument that produces a broad variety of timbres and pitches that sound similar to the Ondes Martenot and Trautonium. Beyond specific construction and design details of the Phantastron, the instrument is also presented as a method for a DIY practice encompassing repurposing, redesigning, hacking, and circuit bending upon a historical design. The works of Paul DeMarinis, Nicolas Collins and Daniel Wilson amongst others are discussed in relation to artists appropriating vintage technology. Parker concludes by considering some of the myriad of possibilities for hybridising digital technology with antique concepts in electronics.

Steve Jones takes Barbara Ballard's 'Carry Principle' that defines the core elements of a mobile experience as a starting point for considering readily available mobile devices such as tablet computers and smartphones as folk instruments. From this starting point, Jones argues the emergence of new forms of mobile music that could be considered folk. He uses Robert Ashley's Music with Roots in the Aether (a series of video portraits of celebrated American composers) as a key reference. In this video Ashley and Gordon Mumma discuss what defines a folk instrument, and they conclude that a characteristic of a folk instrument may include something that is mass-produced and that anybody can use. Ashley and Mumma then present the analogy of the racing bicycle as a folk instrument. Jones goes on to discuss the specific use of the iPad as a platform for mobile music making and the notion of feature creep (the constant addition and expansion of software features) and the impact of this in relation to music making. The issue of toy versus instrument is also discussed.

Gabriel Paiuk's article argues that diverse approaches towards working with electronic sound and the resurfacing of older technologies coupled with digital technologies have created a shift in focus from the creation of novel electronic sounds to the use of electronic sounds as cultural signifiers. From this point of view, he emphasises that cultural traces and codes inherent in the practices and characteristics of media are important considerations in reassessing ways of listening. Paiuk concludes by affirming that, through the use of media appropriation, it is not just a question of technologically produced sound, but sound that operates on a level of 'aural culture'.

Filipe Calegario et al discuss how the growth of DIY culture has led to the emergence of new Digital Musical Instruments (DMIs). The main focus of the article is the cyclic process of design-implementation-evaluation, and the authors present the Sketchument: a software environment devoted to help non-technical users to easily prototype digital musical instruments. Calegario and colleagues consider the use of the ubiquitous mobile device touch screen as a musical interface and the implementation of Sketchument for iPad. Using Neves' competitor analysis, similar tools ranging from Audulus to Reactable Mobile are discussed.

Finally the issue concludes with an off-theme article by Rajmil Fischman that describes the Manual Actions Expressive System (MAES) to enable the creation of music through hand gestures. A P5 Glove is used to track hand and finger positions and as a means to capture data required to devise convincing gestures. A software user interface is implemented in Max/MSP that covers a range of sound processing and manipulation possibilities as well as mapping mechanisms. Fischman is primarily concerned with developing a tool for composition and performance that preserves strong links between the causality of gestures and everyday experience of the world.

This special thematic issue, Re-wiring Electronic Music, points beyond the halcyon days of DIY electronics and practical electronics of the 1960s, the experimental electronic circuits of David Tudor, the objet trouvé and sound object, punk aesthetics and the more recent developments of circuit bending and design, towards a new paradigm within electronic music that combines DIY principles and historical and archaeological views of technology. This field of research and practice has grown in international significance, of which this issue of Organised Sound is testimony with contributions from four continents. I would like to thank Leigh Landy and the Cambridge University Press for devoting an issue to this theme. Also, I would like to thank the reviewers for their time given in the preparation of this issue.

John Richards jrich@dmu.ac.uk 